\title{
Oncofetal proteins in autoimmune- like hepatitis induced by herbals: a
} case report

\author{
Radosveta Tomova ${ }^{1}$, Marija Pishmisheva ${ }^{2}$, \\ Radina Ivanova', Deian Jelev', Krasimir \\ Antonov ${ }^{1}$, Zahariy Krastev'.
}

1. Clinic of Gastroenterology, St. Ivan Rilsky University Hospital, Sofia, Bulgaria;

2. Infectious Department, Hospital, Pazardgik, Bulgaria;

\begin{abstract}
We present a 56-years-old patient with a clinical and biochemical picture of acute hepatitis, but for the fourth time in her life after an eighteen- years period with normal liver tests. The patient had also had concomitant increase in alpha- fetal protein (AFP). After exclusion of viral hepatitis, hemochromatosis and liver cancer, we assumed the diagnosis of autoimmune-like hepatitis, probably caused by a twoyears intake of herbals - Samento and Echinacea. The conducted image investigations did not fiound cancer, despite the increased vitamin B12 level and carbohydrate antigen CA19-9. The histological pattern of damage resembled autoimmune cirrhosis, without convincing biological data for autoimmune hepatitis and clinical signs of cirrhosis. Treatment with corticosteroids and imuran (azathioprine) led to normalization of liver transaminases, vitamin B12 and ferritin levels. Increased levels of AFP, usually slight, haved been reported in acute viral and autoimmune hepatitis, and during Echinacea intake. Elevated CA 19-9 have alsod been also reported in single cases of autoimmune hepatitis and in benign conditions. In our patient both tumour markers were increased, most likely due to the autoimmune-like hepatitis, induced by the taken herbals. After initiation of therapy, AFP quickly reached the normal level, while CA 19-9 decreased slowly during the follow-up. One year later the liver histology was similar to the initial one.
\end{abstract}

Keywords: autoimmune hepatitis, tumor markers, herbals 


\section{Background}

Drug induced liver injury (DILI) requires a wide differential diagnosis and can be difficult to distinguish from autoimmune hepatitis (AlH). Many drugs can awaken latent autoimmunity and lead to drug- - induced autoimmune liver disease (DIAILD), marked by acute or chronic liver injury, autoantibodies, hepatocellular pattern of transaminase elevations, and histology suggestive of idiopathic autoimmune hepatitis. In clinical practice DIAILD resembles idiopathic autoimmune hepatitis. Usually, drug discontinuation leads to improvement, but it may be slow and may require corticosteroid treatment. The time to onset of drug induced autoimmune hepatitis is usually more than 6 months after initiation, but can be more rapid, resembling acute hepatitis. It is difficult to prove that a drug is the cause of the autoimmune hepatitis - like syndrome. Medications that can cause autoimmune hepatitis include minocycline, nitrofurantoin, hydralazine, methyldopa, statins, fenofibrate, alpha and beta interferon, infliximab, etc. Case reports haved also implicated many other agents and herbals - Amanita species, "Bush" herbal teas, Chaparral, Comfrey, Echinacea, Senna fruit extracts, Valerian and others (1, 2).

Increased tumor markers should be interpreted carefully, on the basis of additional investigations. Elevated CA 19-9 may be found in benign and malignant diseases - pancreatic cancer, hepato-biliary, colon and gastric cancer, sometimes without obvious reason (3). Serum AFP can increase in hepatocellular carcinoma, seminoma, germ cell tumors, gastric, biliary, pancreatic cancer, and metastatic liver disease $(4,5)$. Elevated AFP may also be observed in benign liver diseases such aslike acute and chronic viral hepatitis, autoimmune hepatitis and liver cirrhosis (5).

We present a case of an autoimmune-like hepatitis with concomitant tumor markers increase, without malignancy, most likely induced by the takieng of herbals.

\section{Case description}

Acute liver injury at presentation: A 56-year-s old female patient had significantly increased bilirubin and transaminases after a respiratory infection in January 2014 and antibiotic treatment with amikacin ( figure 1). 


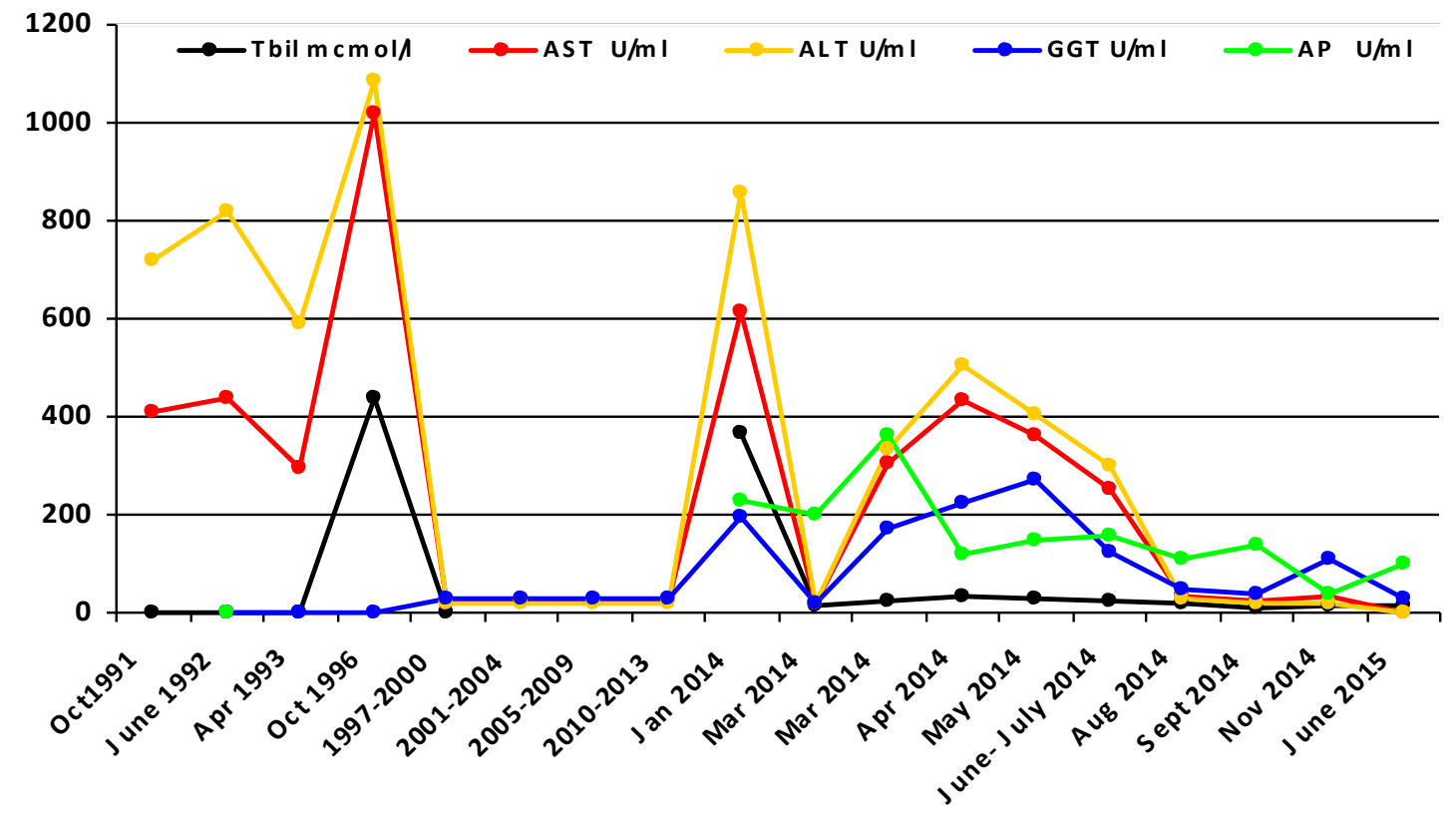

Figure 1. Four time flare of bilirubin and transaminases in a 56- year-s old woman.

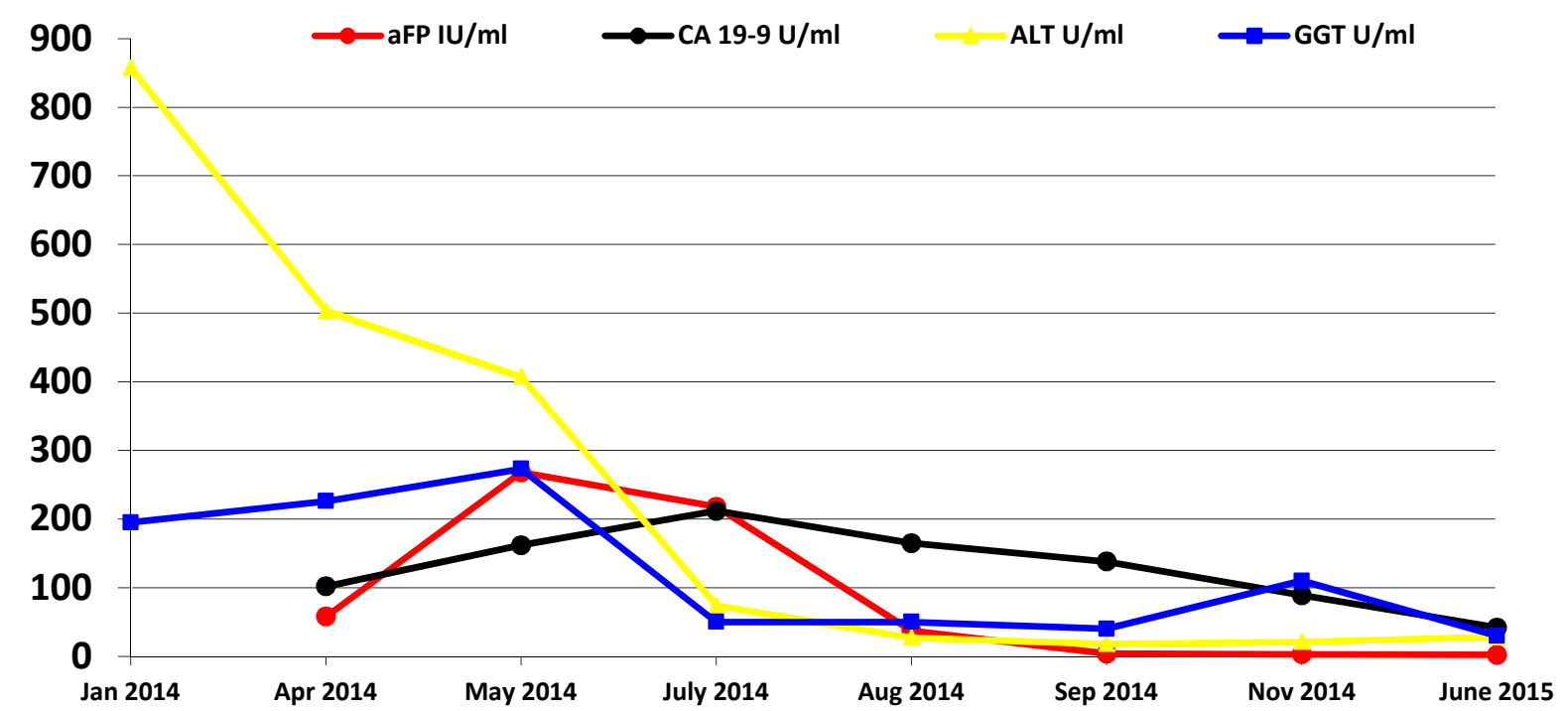

Figure 2. Oncofetal proteins in a 56- year-s old woman with herbals- induced autoimmune-like hepatitis 
High levels of AFP and CA19-9 were also found (figure 2). Elevations in serum Vitamin B12 level - 955,6 $\mathrm{pmol} / \mathrm{l}$ (normal range 141-489) and ferritin $-848 \mathrm{ng} / \mathrm{ml}$ (normal range 13-150) were registered. Hepatitis etiology and patient history: Hepatitis A, B, C, D and E were excluded. The patient had viral hepatitis A in childhood; acute non-A, non-B hepatitis in 1991 with positive anti-HCV antibodies in 1992 (after therapy with levamisole in 1994 anti-HCV and HCV RNA were negative); acute hepatitis in 1996 with negative tests for HCV and HBV. FromAfter 1997 until January 2014 the patient had normal transaminases during follow- up.

Iron homeostasis parameters: Additional tests showed a high serum iron concentration, increased ferritin levels, normal iron binding capacity, and transferrin saturation (TS) near upper normal limit, and also positive oral glucose tolerance test (diabetes). These findings required a differential diagnosis with hemochromatosis. (figure 3 and 4).

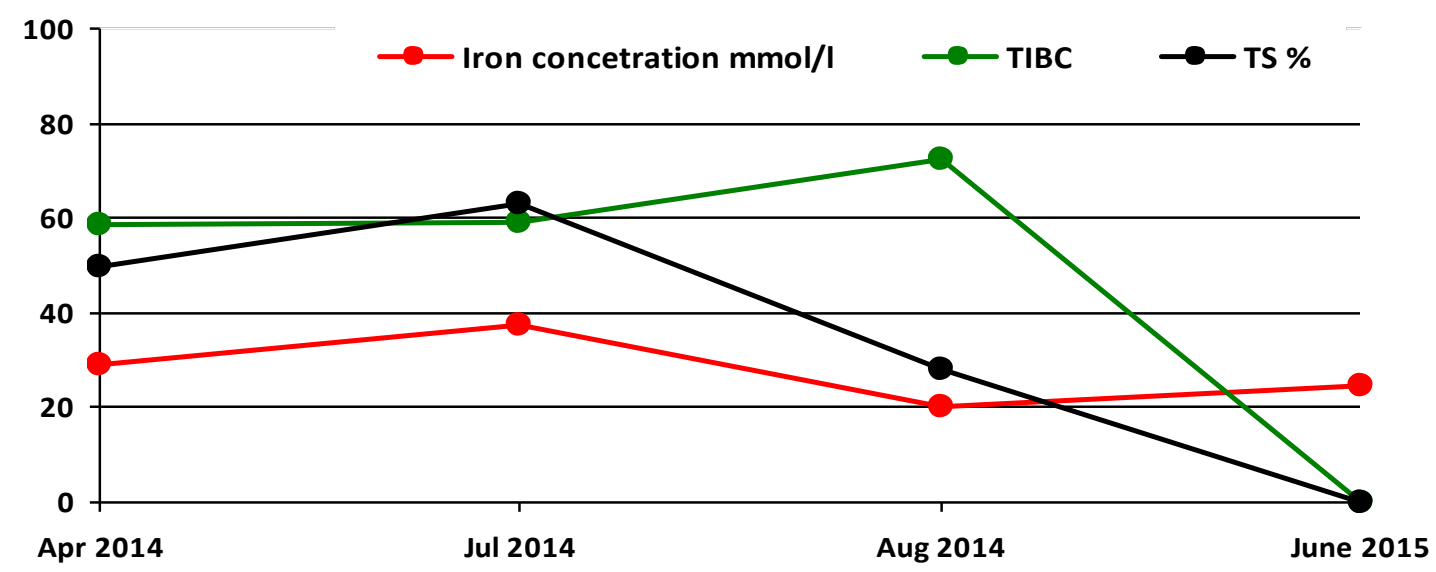

Figure 3. Biomarkers of iron homeostasis in a 56- year-s old woman with herbals- induced autoimmune-like hepatitis

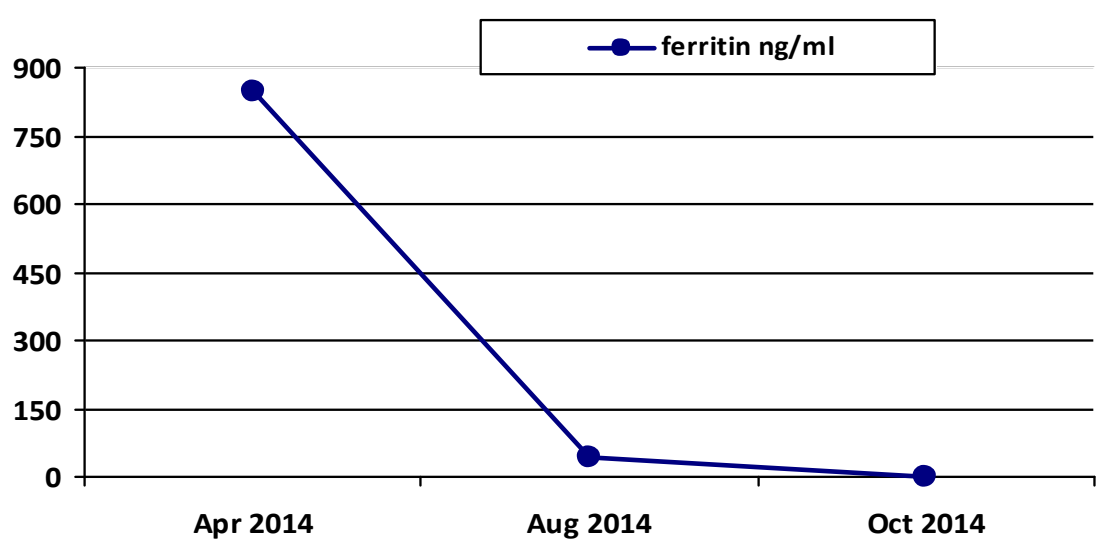

Figure 4. Ferritin levels in a 56- year-s old woman with herbals- induced autoimmune-like hepatitis 
Markers for autoimmune hepatitis: A slightly increased level of IgG - 20,53 $(6,58-18,37)$ with pANCA 1:160 (normal range < 1:20) were found, but Ig A, Ig M, ANA, AMA, anti-LKM, ASMA, LC-1, SLA/LP, Sp100, Ro52, Gp210, 3EBPO were negative. The revised diagnostic score for autoimmune hepatitis was 10 points, making the diagnosis of autoimmune hepatitis probable but not certain.

Additional information: After a detailed and insistent interview the patient reported regular use of echinacea and samento - cat's claw (fromsince 2012 until January 2014) and frequent use of non-steroid anti-inflammatory drugs due to viral respiratory infections. She had also intermittently taken remifemin (Cimicifugae rhizomea extra) for postmenopausal symptoms, and cetaloft for depression.

Oncofetal proteins - differential diagnosis: Many ultrasound examinations, contrast computer tomography (CT) and magnetic resonance (MR) cholangiography finrom May 2014 excluded hepatic, biliary or other tumor and did not reveal pathologic changes in bile ducts. A peripapillary duodenal diverticulum was found, confirmed by gastroscopy.

Histology: Liver histology infrom July 2014 showed an autoimmune type of liver damage with significant portal inflammation, interface hepatitis, and bridging necrosis. There were many lymphocyte infiltrates intralobularly and hepatocytes with balloon degeneration, slight steatosis (10\%) and many fibrotic septaforming single pseudonodules. We accepted an autoimmune-like disease, probably induced by the used herbals (figure 5).

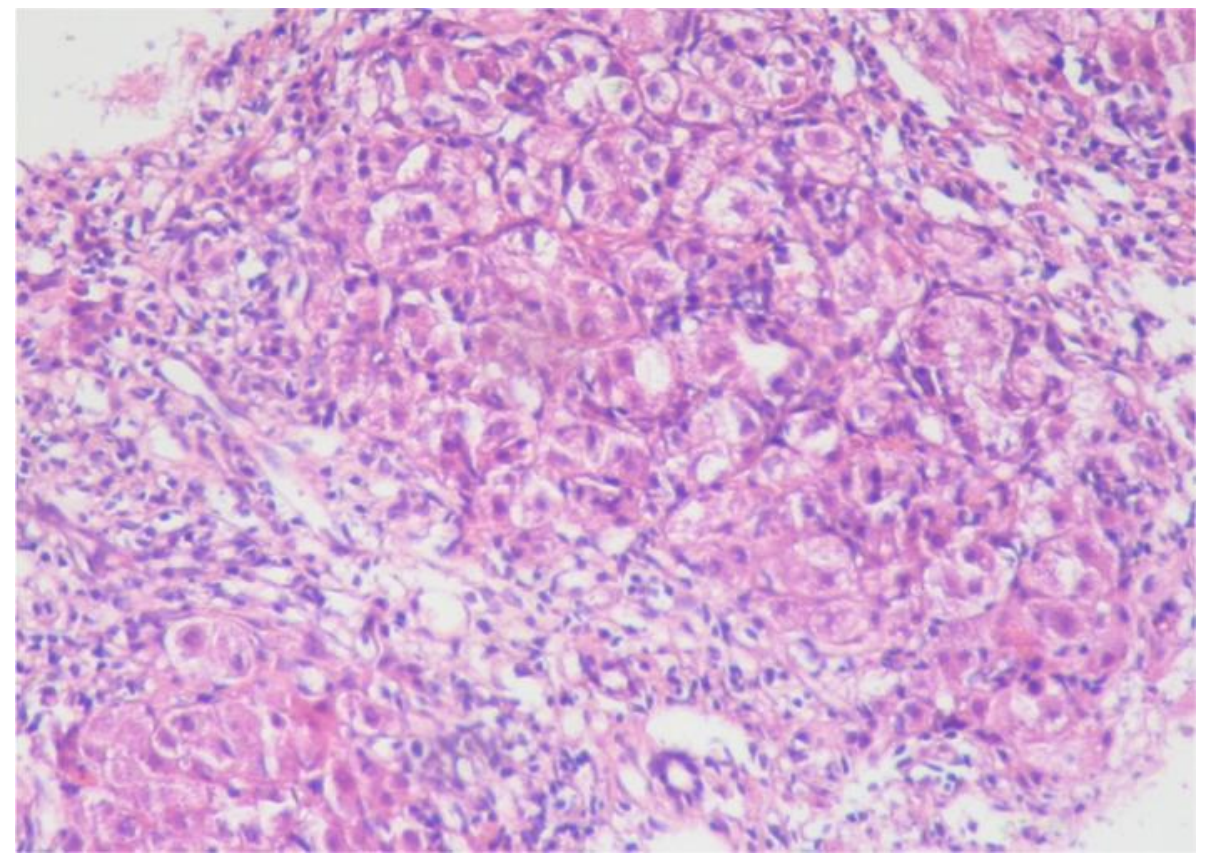

Figure 5. Liver histology ofin a 56- year-s old woman with herbals- induced autoimmune-like hepatitis, showing severe portal inflammation and interface hepatitis, intralobular lymphocyte infiltrates and hepatocytes with balloon degeneration (40x, HE)

Treatment and outcome: Immunosuppressive therapy with imuran $100 \mathrm{mg} / \mathrm{d}$ and low-dose dehydrocortison (15 mg/d), fordue to the diagnosed diabetes, was commenced. A month later a marked 
improvement with a drop in bilirubin and transaminase levels, reaching normal levels, was observed. During follow-up AFP and ferritin normalized and CA 19-9 showed a tendency to decrease, but remained slightly elevated. The cCorticosteroid therapy continued till March 2015 and thereafter the patient received only imuran.

Reassessment of liver histology in September 2015 revealed persistent chronic autoimmune hepatitis with moderate activity and stage of fibrosis F3-F4 (figure 6).

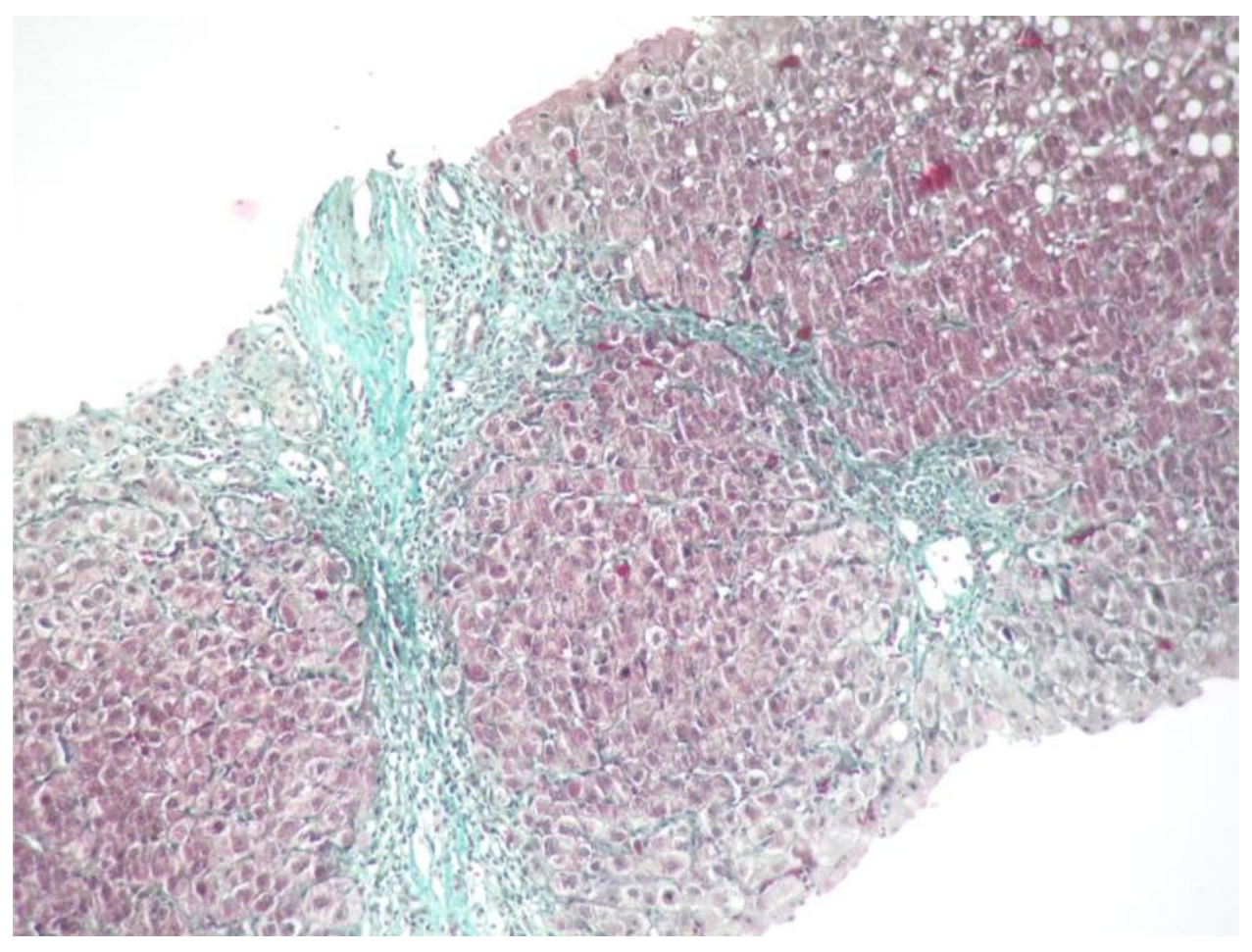

Figure 6. Liver histology ofin a 56- year-s old woman with herbals- induced autoimmune-like hepatitis after immunosuppressive therapy, showing moderate inflammation activity and stage of fibrosis F3-F4 (40x, Masson)

\section{Discussion}

\section{Autoimmune hepatitis and drugs}

AlH presents with elevated transaminases, minimal or normal alkaline phosphatase, hypergammaglobulinaemia with increased immunoglobulin $G(\lg G)$, female predominance, high titers of autoantibodies, immunogenic background, concomitant autoimmune diseases, and good response to immunosuppressive treatment. Our patient had only slightly elevated IgG and pANCA, while the other autoantibodies were negative. No concomitant autoimmune disease was present. Elevated pANCA are characteristic offor primary sclerosing cholangitis and microscopic polyangitis, may be present in inflammatory bowel disease and according to some data in AlH type 1 (6). Magnetic resonance 
cholangiography and histology in our patient excluded bile-duct pathology. All these facts, along with a borderline score for autoimmune hepatitis, made this diagnosis uncertain. Exclusion of viral infection, Wilson's disease and hemochromatosis, along with the histology, confirmed the presence of an autoimmune damage. The patient had a history of anti-HCV positive antibody in 1992 and consequent episodes of elevated bilirubin and transaminases in the past, without elucidated reason. This was followed by a long period of normal liver biochemical tests until January 2014. (Ffigure 1). So, taking in consideration this information, several possibilities were discussed: 1. reactivation of preexisting $\mathrm{AlH} ; 2$. autoimmune disease, awakened by the used drugs; 3 . all is coincidental.

When a DIAILD is suspected several possibilities exist:

First - reactivation of a known AlH upon a new drug introduction (AlH with DILI), though it is difficult to demonstrate a causal relationship. Often, there is advanced fibrosis ion histology.

Second - triggering of the disease in genetically predisposed persons (drug-induced autoimmune hepatitis-DI-AlH). This scenario may lead to a chronic process with a permanent need of immunosuppression.

Third - immune-mediated drug- induced liver injury (IM-DILI) - acute or chronic, depending on the drug exposure duration. In such situations the disease resolves or becomes quiescent with drug withdrawal. Additional symptoms may include fever, eosinophilia, lymphadenopathy or rash and are indistinguishable from true $\mathrm{AlH}$. This type of adverse drug reaction, which can be viewed as autoimmune hypersensitivity, is the most frequent one.

And probably a fourth type with mixed features must be added (7).

In our case both first and second scenarios are possible. The more frequentoften use of natural medicine and herbal remedies increases liver toxicity risk and the possibility of unwanted drug interactions.

Clinical trials with remifemin (Cimicifuga rhizomea extract), used for postmenopausal symptoms relief, had demonstrated a safe profile with the possibility of hepatotoxic reactions. $(6,8)$. A recent report concludes that there was no evidence to support the presumed liver damage (9).

Echinacea contains pyrrolizidine alkaloids (PA), which explains the associated hepatotoxicity associated. This herbal is used as immune booster for common cold and viral infections. Though no significant hepatotoxicity of Echinacea hasd been reported (2), except single cases, the potential effect of this drug in triggering autoimmune diseases in predisposed persons or in combination with other agents is unknown.

Samento (cat's claw), claimed as a potential immune booster, recommended for use in infections, arthritis, inflammatory bowel disorders and cancer, has also no evident hepatotoxicity (10).

These data are inconclusive. There is no information concerning the long-term use of these agents and the cumulative effect of concomitant use of different immunomodulators or potentially hepatotoxic drugs. The question regarding a potential triggering of an autoimmune disease during their prolonged use is still unanswered, and we presume that in our case these herbals could be the trigger for an autoimmune liver disease. 
Cetaloft (citalopram) is a selective reuptake serotonin inhibitor, used as antidepressant. It has a better safety profile, but liver damage is possible. The mechanism of liver injury is unknown, but may be mediated by toxic metabolism intermediates. Cholestatic and hepatocellular patterns of enzyme elevations haved been described. Autoimmune (autoantibodies) and immunoallergic features (rash, fever, and eosinophilia) are uncommon. Recovery is usually rapid once the agent is stopped (11).

The patient had also periodically taken periodically NSAID in low doses. Their use in such pattern is presumed to be safe. Interactions of these drugs with the above mentioned herbals are possible.

\section{AFP and CA 19-9 in liver pathology}

Elevated serum AFP and CA 19-9 in our case required exclusion of a tumour. We excluded such pathology. Obviously, the elevated AFP was due to the autoimmune-like hepatitis and correlated with its severity. Proof for this hypothesis is the normalization of AFP along with liver transaminases during immunosuppressive treatment. CA 19-9 also showed a tendency for decrease, but remained above the normal range.

Oncofetal proteins were studied in various non-neoplastic liver diseases - acute and chronic hepatitis, autoimmune hepatitis, and liver cirrhosis. Several possibilities for increased AFP and CA 19-9 have beenwere reported in the literature besides liver cancer. Increased AFP is registered in autoimmune hepatitis, liver cirrhosis and acute liver failure $(5,12)$. A hHigh AFP level $(>10 \mathrm{ng} / \mathrm{mL})$ may indicate recovery through active liver regeneration or the early recovery within 2 weeks in acute hepatitis $A(13)$. The hHigher level was not associated with improved outcome (14). Increased CA 19-9 was described in autoimmune hepatitis (15-17), with decrease during corticosteroid treatment. Elevation in CA 19-9 was also registered in significant percentage of patients with chronic HCV and HBV hepatitis (40\%), and in patients with cirrhosis (50\%) (18). Patients with acute hepatitis had higher levels of CA 19-9, compared to chronic hepatitis and cirrhosis (19).

Though CA $19-9$ is associated with gastrointestinal cancer, additional data point that significant elevations (greater than $129 \mathrm{U} / \mathrm{mL}$ ), do not always correlate with cholagiocarcinoma in primary sclerosingating cholangitis (20). CA 19-9 can increase in different benign conditions, including diverticular disease, as well $(3,21)$.

Increased serum vitamin B12 may be a sign of several hematologic disorders. Acute hepatitis, cirrhosis, hepatocellular carcinoma and metastatic liver disease can also be accompanied by an increase in this marker. This phenomenon is predominantly caused by cobalamin release during hepatic cytolysis or decreased cobalamin clearance by the affected liver, which was probably the explanation in our patient (22).

AlH, like most inflammatory conditions, is associated with increased ferritin (acute-phase reactant) and typically with near-normal transferrin saturation - such changes were observed in our patient. Excessive ferritin level in the absence of high-transferrin saturation helps differentiate secondary iron overload from hemochromatosis, where transferrin saturation is typically high. The reason for this is unclear, but possible mechanisms can be dysregulation of iron homeostasis in enterocytes and release of iron from hepatocytes following cell damage and fibrosis (23). The changes in iron homeostasis in our patient were 
probably connected with the severity of the disease, sinceas long as during follow up these parameters returned to normal.

\section{Conclusion}

The aAutoimmune type of liver disease in our case was probably related to and triggered by the multiple drugs and herbals, concomitantly used for a long time by the patient. It is hard to point out a single remedy responsible for that. Obtaining sufficient information regarding the long-term use of different herbals and their interactions with other drugs, and of course their relationship to the autoimmune diseases, is necessary.

Exclusion of malignancy is obligatory in cases of elevated tumour markers, but it is also important to analyze all possibilities for that.

\section{References}

1. Liu ZX, Kaplowitz N. Immune-mediated drug-induced liver disease. Clin Liver Dis 2002; 6 (3): 755 74.

2. Reem J. Abdualmjid and Consolato Sergi. Hepatotoxic Botanicals - An Evidence-based Systematic Review. J Pharm Pharmaceut Sci 2013 (www.cspsCanada.org) 16 (3): 376-404.

3. Pavai S, Yap SF. The Clinical Significance of Elevated Levels of Serum CA 19・9. Med J Malaysia 2003; 58 (5):; 667-672.

4. van der Veek PP1, de Vos Tot Nederveen Cappel WH, Langers AM, van Hoek B. Two Patients with Extremely Elevated Tumor Markers: Where Is the Malignancy? Gastroenterol Res Pract. 2011; 2011: 123743. doi: 10.1155/2011/123743.

5. Wójtowicz-Chomicz K1, Cichoz-Lach H, Lis E, Kowalik A, Słomka M. Evaluation of alphafetoprotein concentration in patients with chronic liver diseases. Pol Merkur Lekarski. 2012; 32 (192): 374-377.

6. Naser B1, Schnitker J, Minkin MJ, de Arriba SG, Nolte KU, Osmers R. Suspected black cohosh hepatotoxicity: no evidence by meta-analysis of randomized controlled clinical trials for isopropanolic black cohosh extract. Menopause. 2011;18 (4): 366-375.

7. Castiella A1, Zapata E, Lucena MI1, Andrade RJ. Drug-induced autoimmune liver disease: a diagnostic dilemma of an increasingly reported disease. World J Hepatol 2014; 6 (4): 160-168.

8. Assessment report on Cimicifuga racemosa (L.) Nutt., rhizome. European Medicines Agency. Committee on Herbal Medicinal Products (HMPC) Website www.ema.europa.eu

9. Teschke R., Schwarzenboeck A. Suspected hepatotoxicity by imicifugae racemosae rhizoma (black cohosh, root): critical analysis and structured causality assessment. Phytomedicine. 2009; 16 (1): 72-84. doi: 10.1016/j.phymed.2008.09.009. 
10. Ibrahim KE, Al-Ashban RM, El-Sammani SA. A Study of the Toxicity of Cat's Claw Herbal Medicine. Research Journal of Pharmacology 2009; 3 (3):; 52-57.

11. Clinical and research information on drug-induced liver injury. Citalopram. Overview. United States National Library of Medicine.LiverTox.

12. Yamagiwa S, Tamura $Y$, Takamura $M$, Genda $T$, Ichida $T$, Ishikawa $T$ et al. Increase of fucosylated alpha-fetoprotein fraction at the onset of autoimmune hepatitis and acute liver failure. Hepatol Res. 2014; 44 (14): E368-375. doi: 10.1111/hepr.12318.

13. Seo SI, Kim SS, Choi BY, Lee SH, Kim SJ, Park HW et al. Clinical significance of elevated serum alpha-fetoprotein (AFP) level in acute viral hepatitis A (AHA). Hepatogastroenterology. 2013; 60 (127): 1592-1596.

14. Schiødt FV, Ostapowicz G, Murray N, Satyanarana R, Zaman A, Munoz S. et al. Alphafetoprotein and prognosis in acute liver failure Liver Transpl. 2006 Dec; 12 (12): 1776-1781.

15. Calisto JL, Tagle M, Bedoya P, Scavino Y, Luna E, Poletti L. et al. Autoimmune hepatitis with elevation of CA 19-9 and normalization with immunosuppressant [corrected] treatment: case report and review of the literature. Rev Gastroenterol Peru. 2008; 28 (2): 167-170.

16. Liu FC, Chang DM, Lai JH, Lin CK, Chen HC, Hou TY et al. Autoimmune hepatitis with raised alpha-fetoprotein level as the presenting symptoms of systemic lupus erythematosus: a case report. Rheumatol Int. 2007; 27 (5): 489-491.

17. Montalto M., Santoro L, Vastola M, Curigliano V, Ricci R., Vecchio FM. et al. Normalisation of high CA 19-9 values in autoimmune hepatitis after steroidal treatment. Int $\mathrm{J}$ Immunopathol Pharmacol. 2005; 18 (3): 603-607.

18. Bertino G, Ardiri AM, Calvagno GS., Malaguarnera G., Interlandi D., Vacante M. et al. Carbohydrate 19.9 Antigen Serum Levels in Liver Disease BioMed Research International Volume 2013 (2013), Article ID 531640, 6 pages.

19. Fabris C., Falleti E., Pirisi M., Soardo G., Toniutto P., Vitulli D. et al. Non-specific increase of serum carbohydrate antigen 19-9 in patients with liver disease associated with increased circulating levels of adhesion molecules. Clin Chim Acta. 1995 Dec 15; 243 (1): 25-33.

20. Sinakos E, Saenger AK, Keach J, Kim WR, Lindor KD. Many patients with primary sclerosing cholangitis and increased serum levels of carbohydrate antigen 19-9 do not have cholangiocarcinoma. Clin Gastroenterol Hepatol. 2011; 9 (5): 434-439.

21. Ventrucci M, Pozzato P, Cipolla A, Uomo G. Persistent elevation of serum CA 19-9 with no evidence of malignant disease. Dig Liver Dis 2009; 41: 357-363.

22. Ermens AA, Vlasveld LT, Lindemans J. Significance of elevated cobalamin (vitamin B12) levels in blood. Clin Biochem. 2003 Nov; 36 (8): 585-590. 
23. An IC1, Tiwari AK, Ameda S, Laird-Fick HS. Autoimmune Hepatitis: Diagnostic Dilemma in the Setting of Suspected Iron Overload. Case Rep Gastrointest Med. 2013; 2013: 872987. doi: $10.1155 / 2013 / 872987$.

\section{Corresponding author:}

Radosveta Tomova

Clinic of Gastroenterology, St. Ivan Rilsky University Hospital

15, Acad. Ivan Geshov Blvd., 1431 Sofia, Bulgaria

Phone: +359 2952 6319, Fax: +3592 8510615

e-mail: raditomova@yahoo.com 